\title{
Dysfunctional fatty acid oxidation in renal fibrosis
}

Accumulation of lipid in tubular epithelial cells has been proposed to have a pathogenic role in the development of renal fibrosis. New findings, however, indicate that dysfunctional fatty acid oxidation rather than intracellular lipid accumulation per se induces the development of renal fibrosis.

Fibrosis is a final common pathway in various forms of chronic kidney disease (CKD). To investigate common cellular pathways involved in the development of fibrosis, Katalin Susztak and colleagues performed genome-wide transcriptome analyses of 95 microdissected human kidney samples from patients with diabetic or hypertensive CKD and from individuals with normal kidney function. Their analyses identified several metabolic pathways that were altered in the CKD samples; levels of genes related to fatty acid metabolism and glucose oxidation, in particular, were considerably lower in samples from patients with CKD than in those from healthy controls. The lower levels of fatty acid oxidation were associated with an accumulation of lipid in tubular epithelial cells. Similarly, mice with either genetically induced renal fibrosis or folic acid-induced nephropathy had lower transcript levels of key enzymes involved in fatty acid oxidation, including the transcriptional regulators Ppara and Ppargc 1a, than did control mice.

To examine the potential role of lipid accumulation in fibrosis, Susztak and colleagues generated a transgenic mouse that overexpressed the long chain fatty acid transporter CD36, specifically on tubule cells. "We found evidence of lipid accumulation in these transgenic animals; however, they did not develop significant fibrosis, indicating that lipid accumulation may

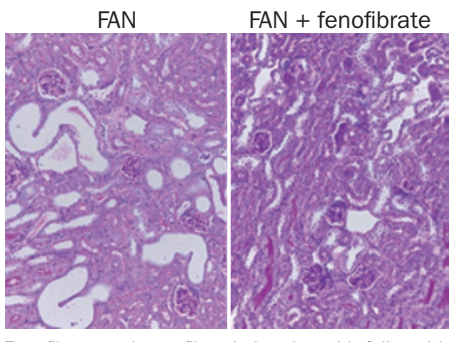

Fenofibrate reduces fibrosis in mice with folic acidinduced nephropathy (FAN). Permission obtained from Nature Publishing Group @ Kang, H. M. et al. Nat. Med. doi: 10.1038/nm.3762

only have a minor role in fibrosis development," she explains. The researchers then tested the effects of restoring fatty acid oxidation on tubulointerstitial fibrosis.

"Transgenic overexpression of the transcription factor Ppargc 1a protected mice from lipid accumulation and fibrosis without restoring the defect in glucose metabolism," says Susztak. "Likewise, pharmacological activation of carnitine palmitoyltransferase 1 or of Ppara by administration of fenofibrate also reduced fibrosis and improved kidney function, indicating that depressed fatty acid metabolism is critical for the development of renal fibrosis."

Susztak points out that recent clinical trials have shown some benefit of fenofibrate on albuminuria in patients with type 2 diabetes mellitus. "However, fenofibrate interferes with kidney function measurements and it is therefore difficult to determine the exact size of this effect," she says. "We are now interested in developing potential new therapeutics that affect this pathway." She also plans to further characterize the metabolic dysregulation in CKD and its role in regulating epigenetic changes and disease progression.

Susan J. Allison

Original article Kang, H. M. et al. Defective fatty acid oxidation in renal tubular epithelial cells has a key role in kidney fibrosis development. Nat. Med. doi:10.1038/nm.3762 